\title{
Spectral sum rules and magneto-roton as emergent graviton in fractional quantum Hall effect
}

\author{
Siavash Golkar, Dung X. Nguyen and Dam T. Son \\ Enrico Fermi Institute, James Franck Institute and Department of Physics, \\ University of Chicago, Chicago, Illinois 60637, U.S.A. \\ E-mail: golkar@damtp.cam.ac.uk, nxdung86@uchicago.edu, \\ dtson@uchicago.edu
}

\begin{abstract}
We consider gapped fractional quantum Hall states on the lowest Landau level when the Coulomb energy is much smaller than the cyclotron energy. We introduce two spectral densities, $\rho_{T}(\omega)$ and $\bar{\rho}_{T}(\omega)$, which are proportional to the probabilities of absorption of circularly polarized gravitons by the quantum Hall system. We prove three sum rules relating these spectral densities with the shift $\mathcal{S}$, the $q^{4}$ coefficient of the static structure factor $S_{4}$, and the high-frequency shear modulus of the ground state $\mu_{\infty}$, which is precisely defined. We confirm an inequality, first suggested by Haldane, that $S_{4}$ is bounded from below by $|\mathcal{S}-1| / 8$. The Laughlin wavefunction saturates this bound, which we argue to imply that systems with ground state wavefunctions close to Laughlin's absorb gravitons of predominantly one circular polarization. We consider a nonlinear model where the sum rules are saturated by a single magneto-roton mode. In this model, the magneto-roton arises from the mixing between oscillations of an internal metric and the hydrodynamic motion. Implications for experiments are briefly discussed.
\end{abstract}

KeYwords: Field Theories in Lower Dimensions, Sum Rules, Effective field theories

ARXIV EPRINT: 1309.2638 


\section{Contents}

1 Introduction 1

2 The effective action 3

2.1 Review of the Newton-Cartan formalism 3

2.2 Physics at the Coulomb energy scale 4

2.3 Gravitational response, spectral representations and shift sum rule 5

$\begin{array}{lll}2.4 & \text { Static structure factor and high-frequency shear modulus } & 6\end{array}$

$\begin{array}{ll}2.5 & \text { Inequalities following from the sum rules }\end{array}$

$\begin{array}{lll}3 & \text { A gravitational model of the magneto-roton } & 8\end{array}$

$\begin{array}{lll}3.1 & \text { Dispersion relation for the magneto-roton } & 9\end{array}$

4 Conclusion $\quad 12$

\section{Introduction}

Fractional quantum Hall (FQH) systems represent the paradigm for interacting topological states of matter. Much attention has been concentrated on the topological properties of the quantum Hall states, encoded in the ground state wavefunction and the statistics of quasiparticle excitations. On the other hand, FQH systems also have a neutral collective excitation known as the magneto-roton. The magneto-roton is an excitation within one Landau level and hence has energy at the interaction (Coulomb) energy scale. The existence of the magneto-roton was suggested in the classic work of Girvin, MacDonald, and Platzman [1], in which Feynman's theory of the roton in superfluid helium is extended to the FQH case. In this picture the magneto-roton is visualized as a long-wavelength density fluctuation. Within the composite fermion theory, the magneto-roton is interpreted as a particle-hole bound state, in which the particle lies in an empty composite-fermion Landau level, and the hole lies in a filled one. In the composite-boson approach, the magneto-roton is interpreted as vortex-antivortex bound state. The magneto-roton has been observed in Raman scattering experiments $[2,3]$ and more recently, in experiments using surface acoustic waves [4]. At nonzero wavenumbers, there is some evidence in favor of more than one magneto-roton mode [5].

Very recently, Haldane has proposed a drastically different interpretation of the magneto-roton [6-8]. He argues that there is a dynamic degree of freedom in FQH systems which can be interpreted as an internal metric. In this picture, the magneto-roton at long wavelength is the quantum of the fluctuations of this metric.

In this paper, we derive some new results related to the physics of a gapped FQH system at the lowest Landau level $(\nu<1)$ at the interaction energy scale. We assume that 
the Coulomb energy is much smaller than the cyclotron energy. First, we derive new, exact sum rules involving the spectral densities of the traceless part of the stress tensor. The two components of the traceless part of the stress tensor are $T_{z z}=\frac{1}{4}\left(T_{x x}-T_{y y}-2 i T_{x y}\right)$, $T_{\bar{z} \bar{z}}=\frac{1}{4}\left(T_{x x}-T_{y y}+2 i T_{x y}\right)$ (here $\left.z=x+i y\right)$, hence we can define two spectral densities:

$$
\begin{aligned}
& \rho_{T}(\omega)=\frac{1}{N} \sum_{n}\left|\left\langle n\left|\int d^{2} x T_{z z}(\mathbf{x})\right| 0\right\rangle\right|^{2} \delta\left(\omega-E_{n}\right), \\
& \bar{\rho}_{T}(\omega)=\frac{1}{N} \sum_{n}\left|\left\langle n\left|\int d^{2} x T_{\bar{z} \bar{z}}(\mathbf{x})\right| 0\right\rangle\right|^{2} \delta\left(\omega-E_{n}\right),
\end{aligned}
$$

where $N$ is the total number of particles in the system, $|0\rangle$ is the ground state and the sums are taken over all excited states $|n\rangle$. Physically, these spectral densities are proportional to the probability that a circularly polarized graviton with energy $\omega$ falling perpendicularly to the system is absorbed. The two functions correspond to the two circular polarizations of the graviton. Without a complete solution to the FQH problem, we do not know $\rho_{T}(\omega)$ and $\bar{\rho}_{T}(\omega)$, but for a gapped FQH system we expect these functions to be zero below a gap $\Delta_{0}$ and to fall to zero when $\omega$ increases far above $\Delta_{0}$. If there is a well-defined magneto-roton at $q=0$, we expect it to appear as peaks in the spectral densities.

We will show that the spectral densities satisfy three sum rules,

$$
\begin{gathered}
\int_{0}^{\infty} \frac{d \omega}{\omega^{2}}\left[\rho_{T}(\omega)-\bar{\rho}_{T}(\omega)\right]=\frac{\mathcal{S}-1}{8}, \\
\int_{0}^{\infty} \frac{d \omega}{\omega^{2}}\left[\rho_{T}(\omega)+\bar{\rho}_{T}(\omega)\right]=S_{4}, \\
\int_{0}^{\infty} \frac{d \omega}{\omega}\left[\rho_{T}(\omega)+\bar{\rho}_{T}(\omega)\right]=\frac{\mu_{\infty}}{\rho_{0}} .
\end{gathered}
$$

In eq. (1.3), $\mathcal{S}$ is the shift of the ground state, defined as the offset in the relationship between the number of magnetic flux quanta $N_{\phi}$, the filling factor $\nu$ and the number of electrons $Q$ when the latter are put on a sphere: $Q=\nu\left(N_{\phi}+\mathcal{S}\right)$ [9]. In eq. (1.4), $S_{4}$ is the coefficient governing the low-momentum behavior of the projected structure factor [1]: $\bar{s}(q)=S_{4}\left(q \ell_{B}\right)^{4}$, where $\ell_{B}$ is the magnetic length. Finally, in eq. (1.5), $\mu_{\infty}$ is the highfrequency elastic modulus, which will be defined exactly later in the text [see eqs. (2.24) and (2.25)], and $\rho_{0}$ is the particle number density in the ground state. In all sum rules, the limit $m \rightarrow 0$ of the spectral densities is taken first, before the upper limit of integration is taken to infinity. In this order of limits, the integral in each sum rule is dominated by $\omega$ of the order of the Coulomb energy.

The sum rule (1.3) is particularly interesting, as it establishes a connection between a topological characteristic of the ground state (the shift) and dynamic information (the spectral densities). In the $\nu=1$ integer quantum Hall state, the sum rule becomes trivial, as there is no degree of freedom at the interaction energy scale (hence $\rho_{T}=\bar{\rho}_{T}=0$ ), and the shift is $\mathcal{S}=1$, so both sides of the sum rule vanish.

Using the three sum rules, we derive some inequalities between different observables in the FQH states. One of these inequalities, previously derived by Haldane [6], places a 
lower bound on the coefficient of the $q^{4}$ asymptotics of the projected static structure factor, which is saturated by the Laughlin's trial wavefunction.

We will also consider a simple model where the sum rules are saturated by one magnetoroton mode, which manifests as the oscillation of the internal metric of the fluid mixed with the hydrodynamic fluid motion. The model provides a concrete realization of Haldane's idea of an internal metric degree of freedom in FQH systems [6]. This model is not meant to be exact, however it does exhibit some of the characteristic properties of the observed magneto-roton modes. We discuss the polarization properties of the magneto-roton in this model, which may be measurable in future experiments.

\section{The effective action}

\subsection{Review of the Newton-Cartan formalism}

Recently, one of the authors has proposed the use of nonrelativistic general coordinate invariance as a way to constrain the dynamics of quantum Hall systems [10] (for related work, see refs. [11, 12]). Although the method can be thought of as "gauging" the Galilean invariance, the local symmetry remains nontrivial in the limit of zero bare electron mass, and hence is a symmetry intrinsic to the physics of electrons at the lowest Landau level, coupled to electromagnetism and gravity.

In ref. [10], the attention was focused on the regime of long wavelengths (much larger than the magnetic length) and low frequencies (much smaller than the gap). In this paper we will relax the latter condition, allowing for energies comparable to the gap. In this regime, terms with arbitrary number of time derivatives must be taken into account in the effective action. However, as we will demonstrate, we can still obtain nontrivial relationships by expanding in the number of spatial derivatives.

We briefly review the main result of ref. [10] here. The effective Lagrangian describing the response of a gapped quantum Hall state to external electromagnetic $\left(A_{0}, A_{i}\right)$ and gravitational perturbations $\left(h_{i j}\right)$, in the massless limit, is:

$$
\mathcal{L}=\frac{\nu}{4 \pi} \varepsilon^{\mu \nu \lambda} a_{\mu} \partial_{\nu} a_{\lambda}-\rho v^{\mu}\left(\partial_{\mu} \varphi-\tilde{A}_{\mu}-s \omega_{\mu}+a_{\mu}\right)+\mathcal{L}_{0}\left[\rho, v^{i}, h_{i j}\right] .
$$

Here $v^{\mu}=\left(1, v^{i}\right) ; a_{\mu}, \rho$ and $v^{i}$ are dynamical fields, with respect to which one should extremize the action; and $\tilde{A}_{\mu}$ is related to the external electromagnetic potential by:

$$
\tilde{A}_{0}=A_{0}-\frac{1}{2} \varepsilon^{i j} \partial_{i}\left(h_{j k} v^{k}\right), \quad \tilde{A}_{i}=A_{i},
$$

$\omega_{\mu}$ is the spin connection of the Newton-Cartan space $\left(h_{i j}, v^{i}\right)$, defined through the derivatives of the vielbein $e_{i}^{a}\left(h_{i j}=e_{i}^{a} e_{j}^{a}\right)$ :

$$
\omega_{0}=\frac{1}{2} \epsilon^{a b} e^{a j} \partial_{0} e_{j}^{b}+\frac{1}{2} \varepsilon^{i j} \partial_{i}\left(h_{j k} v^{k}\right), \quad \omega_{i}=\frac{1}{2} \epsilon^{a b} e^{a j} \nabla_{i} e_{j}^{b} .
$$

Finally, $\mathcal{L}_{0}$ contains all "non-universal" terms, i.e., terms that cannot be fixed by symmetry arguments alone. 
There are two parameters that enter the Lagrangian (2.1): $\nu$, which is identified with the filling factor, and $s$, identified with the orbital spin (per particle) and is related to the shift by $s=\mathcal{S} / 2$. In ref. [10] it was found that these two parameters control some quantities, most notably the $q^{2}$ part of the Hall conductivity at zero frequency ( $q$ being the wavenumber of the perturbation).

\subsection{Physics at the Coulomb energy scale}

It was also found in ref. [10] that most physical quantities, e.g., the same $q^{2}$ part of the Hall conductivity, but calculated at nonzero frequency, are not fixed by $\nu$ and $s$ alone. The same is true for the $q^{4}$ term in the density-density correlation function. Physically, these quantities depend crucially on the physics happening at the Coulomb energy scale $\Delta$. The physics of the gapped excitations is contained in the non-universal part of the Lagrangian $\mathcal{L}_{0}$.

Terms in $\mathcal{L}_{0}$ can be organized in a series over powers of derivatives. We will be interested in the physics at long wavelengths, $q \ell_{B} \ll 1$. The expansion parameter in frequency would be $\omega / \Delta$, however since we are interested in physical phenomena at the scale $\Delta$, we need to keep terms to all orders in time derivatives.

A consistent power counting scheme is to consider fluctuations of the metric $h_{i j}$ and the gauge potentials $A_{0}, A_{i}$ as $O(1)$, and expand in powers of the spatial derivatives. In this work, we will be interested only in the response of the quantum Hall systems to unimodular metric perturbations, i.e., those in which the perturbed metric $h_{i j}$ has determinant equal to one, and to perturbations of the scalar potential $A_{0}$, i.e., perturbations corresponding to a longitudinal electric field, without changing the magnetic field. In this case, the lowest non-trivial terms entering $\mathcal{L}_{0}$ are $O\left(q^{4}\right)$. These we parameterize, without loss of generality, ${ }^{1}$ with two functions $F(\omega)$ and $G(\omega)$. In the following equation the frequency $\omega$ is replaced by $i v \cdot \nabla$ where $\nabla$ is the Newton-Cartan covariant derivative [10],

$$
\mathcal{L}_{0}=-\frac{\rho}{4}\left[\sigma^{\mu \nu} F(i v \cdot \nabla) \sigma_{\mu \nu}+\tilde{\sigma}^{\mu \nu} G(i v \cdot \nabla)\left(v \cdot \nabla \sigma_{\mu \nu}\right)\right] .
$$

Here $\sigma_{\mu \nu}$ is the traceless part of shear tensor (see [10] for precise definition) and $\tilde{\sigma}^{\mu \nu}$ is defined as:

$$
\tilde{\sigma}^{\mu \nu}=\frac{1}{2}\left(\varepsilon^{\mu \alpha \gamma} h^{\nu \beta}+\varepsilon^{\nu \alpha \gamma} h^{\mu \beta}\right) \sigma_{\alpha \beta} n_{\gamma},
$$

where $n_{\mu}=(1, \mathbf{0})$.

We will work only to quadratic order, hence we only need to know the leading terms in the spatial components of the shear tensor:

$$
\sigma_{i j}=\partial_{i} v_{j}+\partial_{j} v_{i}+\dot{h}_{i j}-\delta_{i j}\left(\partial_{k} v_{k}+\frac{1}{2} \dot{h}\right)
$$

The quadratic part of the Lagrangian is then:

$$
\mathcal{L}_{0}=-\frac{\rho_{0}}{4}\left[\sigma_{i j} F\left(i \partial_{t}\right) \sigma_{i j}+\tilde{\sigma}_{i j} G\left(i \partial_{t}\right) \dot{\sigma}_{i j}\right]
$$

\footnotetext{
${ }^{1}$ As an example, the term $\left(\partial_{i} \rho\right)^{2}$ is of higher order, because fluctuations of $\rho$ are of order $q^{4}$, as evident from eq. (2.17) below. The same is true for $\left(\partial_{i} v^{i}\right)^{2}$ : because of charge conservation $\partial_{i} v^{i} \sim \partial_{t} \rho \sim q^{4}$.
} 
Here, for any symmetric traceless tensors $A_{i j}$ we define $\tilde{A}_{i j}=\frac{1}{2}\left(\epsilon_{i k} A_{k j}+\epsilon_{j k} A_{k i}\right)$ which is again a symmetric traceless tensor. It is also easy to show $\tilde{\tilde{A}}_{i j}=-A_{i j}$ and $\tilde{A}_{i j} B_{i j}=-A_{i j} \tilde{B}_{i j}$.

\subsection{Gravitational response, spectral representations and shift sum rule}

We now relate the two functions $F(\omega)$ and $G(\omega)$ to the spectral densities of the stress tensor $\rho_{T}$ and $\bar{\rho}_{T}$. The two-point function of the stress tensor can be read directly from the action, and is simplest for the traceless components at zero spatial momentum. After a simple calculation we get:

$$
\begin{aligned}
\langle\bar{T} T\rangle_{\omega} & =-\frac{\omega}{4} s \rho_{0}+\frac{\omega^{3}}{2} \rho_{0} G+\frac{\omega^{2}}{2} \rho_{0} F, \\
\langle T \bar{T}\rangle_{\omega} & =\frac{\omega}{4} s \rho_{0}-\frac{\omega^{3}}{2} \rho_{0} G+\frac{\omega^{2}}{2} \rho_{0} F .
\end{aligned}
$$

The spectral densities defined in eqs. (1.1) and (1.2) are related to $F$ and $G$ by:

$$
\begin{aligned}
& \rho_{T}(\omega)=-\frac{\omega^{2}}{2 \pi} \operatorname{Im}(F+\omega G), \\
& \bar{\rho}_{T}(\omega)=-\frac{\omega^{2}}{2 \pi} \operatorname{Im}(F-\omega G),
\end{aligned}
$$

where we have extended the definition of the spectral densities to negative $\omega$ 's by requiring $\rho_{T}(-\omega)=\bar{\rho}_{T}(\omega)$. The functions $F(\omega)$ and $G(\omega)$ are regular in the $\omega \rightarrow 0$ limits, and as we shall explain below [see eqs. (2.15) and (2.21)] they should both fall as $1 / \omega^{2}$ when $\omega \gg \Delta$. From these behaviors we can write down the spectral representations of $F$ and $G$ :

$$
\begin{aligned}
& F(\omega)=2 \int_{0}^{\infty} \frac{d \omega^{\prime}}{\omega^{\prime}} \frac{\rho_{T}\left(\omega^{\prime}\right)+\bar{\rho}_{T}\left(\omega^{\prime}\right)}{\omega^{2}-\omega^{\prime 2}+i \epsilon}, \\
& G(\omega)=2 \int_{0}^{\infty} \frac{d \omega^{\prime}}{\omega^{\prime 2}} \frac{\rho_{T}\left(\omega^{\prime}\right)-\bar{\rho}_{T}\left(\omega^{\prime}\right)}{\omega^{2}-\omega^{\prime 2}+i \epsilon} .
\end{aligned}
$$

The Hall viscosity, as a function of frequency, can be related through a Kubo's formula to the parity-odd part of the two-point function of the stress tensor [13]. We find:

$$
\eta_{\mathrm{H}}(\omega)=\rho_{0}\left(\frac{s}{2}-\omega^{2} G(\omega)\right) .
$$

At $\omega \rightarrow 0$ this equation gives the relationship between the (zero-frequency) Hall viscosity and the shift: $\eta_{\mathrm{H}}(0)=\rho_{0} \mathcal{S} / 4$. At frequencies much larger than $\Delta$, interactions can be neglected and the Hall viscosity is determined completely by the Berry phase of each orbital under homogeneous metric deformation. The computation of the high-frequency Hall viscosity (where "high" means frequencies much larger than the Coulomb energy scale, but still much smaller than the cyclotron energy) proceeds in exactly the same way as in the integer quantum Hall case [14], and the result is $\eta_{\mathrm{H}}(\infty)=\rho_{0} / 4$. Thus we find:

$$
\lim _{\omega \rightarrow \infty} \omega^{2} G(\omega)=\frac{\mathcal{S}-1}{4},
$$

and using eq. (2.13) we derive our first sum rule:

$$
\int_{0}^{\infty} \frac{d \omega}{\omega^{2}}\left[\rho_{T}(\omega)-\bar{\rho}_{T}(\omega)\right]=\frac{\mathcal{S}-1}{8} .
$$




\subsection{Static structure factor and high-frequency shear modulus}

We now derive two sum rules involving the sum of the two spectral densities $\rho_{T}(\omega)+\bar{\rho}_{T}(\omega)$. Computing the two-point function of the density from the action from (2.1) and (2.7), we find:

$$
\int d^{3} x e^{i \omega t-i \mathbf{q} \cdot \mathbf{x}}\langle T \rho(t, \mathbf{x}) \rho(0, \mathbf{0})\rangle=i \rho_{0}\left(q \ell_{B}\right)^{4} F(\omega),
$$

Integrating both sides over $\omega$, we get:

$$
\bar{s}(q)=i\left(q \ell_{B}\right)^{4} \int_{-\infty}^{\infty} \frac{d \omega}{2 \pi} F(\omega),
$$

where $\bar{s}(q)$ is the projected static structure factor [1]. The reason we get the projected structure factor instead of the unprojected one is that we are working in the limit of zero band mass, and so when we took the integral over $\omega$, implicitly we have assumed that upper limit of integration is still much smaller than the cyclotron frequency $B / m$. By using eq. (2.12), we find the second sum rule:

$$
\int_{0}^{\infty} \frac{d \omega}{\omega^{2}}\left[\rho_{T}(\omega)+\bar{\rho}_{T}(\omega)\right]=S_{4}
$$

where $S_{4}=\lim _{q \rightarrow 0} \bar{s}(q) /\left(q \ell_{B}\right)^{4}$.

The third sum rule again comes from the stress response at large $\omega$. For this purpose, it is convenient to describe the motion of the fluid in terms of the displacement $u^{i}$, which is related to the velocity by $v^{i}=\dot{u}^{i}$. The $F$ term in the action now reads:

$$
-\frac{\rho_{0}}{4} \int d^{2} x \omega^{2} F(\omega)\left[\partial_{i} u_{j}+\partial_{j} u_{i}+h_{i j}-\delta_{i j}\left(\partial \cdot u+\frac{h}{2}\right)\right]^{2} .
$$

In the limit $\omega \rightarrow \infty$, the $G$ term, having an extra time derivative, does not contribute to the energy. Hence, we are left with only the $F$ contribution above, which takes the exact same form as the the deformation energy of a solid, with the shear modulus $\mu_{\infty}$ given by:

$$
\mu_{\infty}=\frac{\rho_{0}}{2} \lim _{\omega \rightarrow \infty} \omega^{2} F(\omega)
$$

Using the spectral representation of $F(\omega)$ in (2.12), we find:

$$
\int_{0}^{\infty} \frac{d \omega}{\omega}\left[\rho_{T}(\omega)+\bar{\rho}_{T}(\omega)\right]=\frac{\mu_{\infty}}{\rho_{0}} .
$$

The high-frequency shear modulus $\mu_{\infty}$ was introduced in the phenomenological model of refs. [15-17]. We now give the precise meaning of this constant. From our discussion, we know that $\mu_{\infty}$ characterizes the stress response of the system under uniform metric perturbations with frequencies much higher than the Coulomb energy scale, but much lower than the cyclotron energy. Since at these frequencies the Coulomb interaction between electrons can be ignored, each particle evolves independently under such a perturbation. The orbital of each electron is continuously deformed and projected down to the lowest Landau level. In this way, we can completely determine the wavefunction of the deformed 
state from that of the the ground state. For example, consider a metric perturbation in which the $x$ coordinate is stretched by a factor of $e^{\alpha / 2}$ while the $y$ coordinate compressed by $e^{-\alpha / 2}$. If we denote the ground state wave function as:

$$
\Psi\left(z_{i}\right)=f\left(z_{i}\right) \exp \left(-\sum_{i}\left|z_{i}\right|^{2} / 4 \ell_{B}^{2}\right)
$$

the deformed state $\left|\Psi_{\alpha}\right\rangle$ is obtained by replacing $f\left(z_{i}\right)$ by $f_{\alpha}\left(z_{i}\right)$,

$$
\Psi_{\alpha}\left(z_{i}\right)=f_{\alpha}\left(z_{i}\right) \exp \left(-\sum_{i}\left|z_{i}\right|^{2} / 4 \ell_{B}^{2}\right), \quad f_{\alpha}\left(z_{i}\right)=\exp \left[\frac{\alpha}{2} \sum_{i}\left(\ell_{B}^{2} \frac{\partial^{2}}{\partial z_{i}^{2}}-\frac{z_{i}^{2}}{4 \ell_{B}^{2}}\right)\right] f\left(z_{i}\right) .
$$

If we now use Laughlin's wavefunction to substitute for $f\left(z_{i}\right)$ above, the deformed states that we obtain coincide exactly with the ones recently considered in ref. [18].

The energy of these states is a function of $\alpha$ with minimum at $\alpha=0$, and the highfrequency shear modulus is simply the curvature of this function at the minimum:

$$
\mu_{\infty}=\left.\frac{1}{A} \frac{\partial^{2}}{\partial \alpha^{2}}\left\langle\Psi_{\alpha}|\hat{H}| \Psi_{\alpha}\right\rangle\right|_{\alpha=0}
$$

where $A$ is the total area of the system. Equations (2.25) and (2.24) define the constant $\mu_{\infty}$ appearing in the sum rule (2.22).

\subsection{Inequalities following from the sum rules}

The sum rules have important implications. First, since $\rho_{T}$ and $\bar{\rho}_{T}$ are non-negative spectral densities, comparing eqs. (2.16) and (2.19), we obtain the following inequality between $S_{4}$ and $\mathcal{S}$ :

$$
S_{4} \geq \frac{|\mathcal{S}-1|}{8} .
$$

This inequality has been previously derived by Haldane [7, 19]. For Laughlin's fractions $\nu=1 /(2 p+1), \mathcal{S}=1 / \nu$, and the inequality becomes $S_{4} \geq(1-\nu) / 8 \nu$. Remarkably, the Laughlin wavefunction has $S_{4}$ saturating the lower bound. Hence, if the Laughlin wavefunction was the true wavefunction of the ground state, that would imply $\bar{\rho}_{T}=0$ for all $\omega$.

Read and Rezayi [20] argued that the inequality (2.26) is actually an equality for all lowest-Landau-level ground states with rotational invariance. From our derivation, we do not expect the equality to hold generally: the spectral density $\bar{\rho}_{T}$ need not necessarily vanish. Nevertheless, the Laughlin wavefunction seems to be a very good approximation to the true wavefunction of the Coulomb potential, thus it is possible that for the true ground state of the Coulomb problem, $\bar{\rho}_{T}$ is numerically much smaller than $\rho_{T}$.

Finally, we can also put a lower bound on the energy gap $\Delta_{0}$ at $q=0$. The energy gap may correspond not to a single quasiparticle, but, for example, to a pair of magneto-rotons, in which case $\Delta_{0}$ is the start of a continuum. The inequality that follows from comparing the sum rules (2.19) and (2.22) is:

$$
\Delta_{0} \leq \frac{\mu_{\infty}}{\rho_{0} S_{4}}
$$


where equality would be achieved only when $\rho_{T}$ and $\bar{\rho}_{T}$ are proportional to $\delta\left(\omega-\Delta_{0}\right)$. The equality in this case has the same form as the Girvin-MacDonald-Platzman variational formula for the magneto-roton energy, but in contrast to the latter, both the numerator and the denominator in our formula are finite in the limit $q \rightarrow 0$.

By combining these two inequalities we can also write:

$$
\Delta_{0} \leq \frac{8 \mu_{\infty}}{\rho_{0}|\mathcal{S}-1|}
$$

which saturates under the conditions $\bar{\rho}_{T}=0$ and $\rho_{T}(\omega) \sim \delta\left(\omega-\Delta_{0}\right)$.

\section{A gravitational model of the magneto-roton}

We now present a simple model where the sum rules are satisfied by construction and are dominated by one single mode which is identified with the magneto-roton. To start, we adapt the Lagrangian formulation of fluid dynamics [21], in which the degrees of freedom of the quantum Hall fluids are the Lagrangian coordinates $X^{I}(t, \mathbf{x}), I=1,2$. The density and velocity of the fluid are given by:

$$
\rho v^{\mu}=\rho_{0} \varepsilon^{\mu \nu \lambda} \epsilon_{I J} \partial_{\nu} X^{I} \partial_{\lambda} X^{J},
$$

such that the divergence of the current vanishes identically. The theory is required to be invariant under volume-preserving diffeomorphisms in the $X^{I}$ space. Imposing this condition sets the shape modulus to zero, thereby ensuring that our action describes a fluid and not a solid.

The degree of freedom saturating the sum rules, is assumed to be a unimodular metric tensor $G_{I J}$. Physically, one should think of $G_{I J}$ as parameterizing the anisotropic deformation of the ground state, as constructed in section 2.4. In this model, $G_{I J}$ is the only dynamical degree of freedom at the Coulomb energy scale.

The theory can either be written in $x$ space, treating $X^{I}$ as functions of $t$ and $x^{i}$, or in $X$ space, where the dynamical fields are $x^{i}=x^{i}\left(t, X^{I}\right)$. The action of the model is the sum of three parts $S=S_{1}+S_{2}+S_{3}$, where the first part is written in $x$ space:

$$
\mathcal{L}_{1}=\frac{\nu}{4 \pi} \epsilon^{\mu \nu \lambda} a_{\mu} \partial_{\nu} a_{\lambda}+\rho v^{\mu}\left(\partial_{\mu} \varphi-\tilde{A}_{\mu}-\frac{1}{2} \omega_{\mu}+a_{\mu}\right) .
$$

This has the same form as the the universal part of the action derived in ref. [10], but with $s$ replaced by $1 / 2$. The reason for this replacement is that we expect $L_{1}$ to encode the Hall viscosity at high frequency, but not at low frequency.

The second part of the action is written in $X$ space. It is a Wess-Zumino-Witten action:

$$
S_{2}=\frac{\alpha \rho_{0}}{2} \int_{0}^{1} d \tau \int d t d^{2} X \partial_{\tau} G_{I J} G^{J K} \partial_{t} G_{K L} \epsilon^{L I},
$$

where as a function of $\tau, G_{I J}(0, t, X)=\delta_{I J}$ and $G_{I J}(1, t, X)=G_{I J}(t, X)$, and $\alpha$ is a parameter that will be fixed later. Although the action is written as an integral in $\tau$ space, one can check that the action depends only on the boundary value at $\tau=1$, but 
is independent of the interpolation between $\tau=0$ and $\tau=1$. It is identical to the action considered in ref. [22].

Finally, in $S_{3}$ we include the potential energy, which depends on the density and one parameter characterizing the eccentricity of the deformation:

$$
\mathcal{L}_{3}=\mathcal{L}_{3}\left(\varepsilon^{i j} \epsilon_{I J} \partial_{i} X^{I} \partial_{j} X^{J}, G_{I J} h^{i j} \partial_{i} X^{I} \partial_{j} X^{J}\right) .
$$

We only consider small perturbations around the ground state; we take: $X^{I}=x^{I}-u^{I}$ and $G_{I J}=\delta_{I J}+H_{I J}$. Ignoring the constant term, total derivatives and terms proportional to squares of $\partial_{i} u^{i}$ and $h_{i i}$, which are small in the regime we are considering, we have:

$$
\mathcal{L}_{3}=-\frac{\alpha \rho_{0} \Delta}{4}\left(\partial_{i} u_{j}+\partial_{j} u_{i}+h_{i j}-H_{i j}\right)^{2}
$$

Now we introduce the variable $\gamma_{i j}$ as:

$$
\gamma_{i j}=\partial_{i} u_{j}+\partial_{j} u_{i}+h_{i j}-H_{i j}
$$

Using $\gamma$ we can rewrite the quadratic action in the form of eq. (2.1) where $\mathcal{L}_{0}$ is given by:

$$
\mathcal{L}_{0}=\frac{\alpha \rho_{0}}{2}\left(\tilde{\sigma}_{i j} \gamma_{i j}+\frac{1}{2} \tilde{\gamma}_{i j} \dot{\gamma}_{i j}-\frac{\Delta}{2} \gamma_{i j}^{2}\right)
$$

After integrating out $\gamma_{i j}, \mathcal{L}_{0}$ reduces to the form (2.4), with functions $F$ and $G$ given by:

$$
F(\omega)=\frac{\alpha \Delta}{\omega^{2}-\Delta^{2}+i \epsilon}, \quad G(\omega)=\frac{\alpha}{\omega^{2}-\Delta^{2}+i \epsilon} .
$$

This corresponds exactly to the spectral functions $\rho_{T}(\omega) \sim \delta(\omega-\Delta)$ and $\bar{\rho}_{T}=0$. In particular, $\alpha=(\mathcal{S}-1) / 4$ and the inequality $(2.26)$ becomes an equality in this model.

We note here that our model contains a single gapped mode. In our model, the state (2.24) can be thought of a condensate of zero-momentum magnetoroton. The same magneto-roton contributes to all spectral densities, in particular the spectral density of the density operator. In other words, the magneto-roton considered here is the same as the one considered in ref. [1].

\subsection{Dispersion relation for the magneto-roton}

We reiterate the form of the effective Lagrangian in flat space-time and in the massless limit:

$$
\begin{aligned}
\mathcal{L}=\frac{\nu}{4 \pi} \varepsilon^{\mu \nu \lambda} a_{\mu} \partial_{\nu} a_{\lambda}-\rho v^{\mu}\left(\partial_{\mu} \varphi-A_{\mu}+a_{\mu}\right) & +\rho \frac{s-1}{2} \epsilon^{i j} \partial_{i} v_{j} \\
& -\frac{\rho}{4}\left(\sigma_{i j} F(\omega) \sigma_{i j}+\tilde{\sigma}_{i j} G(\omega) \dot{\sigma}_{i j}\right)-\epsilon_{i}(\rho),
\end{aligned}
$$

where $F$ and $G$ are given in eq. (3.8) and the function $\epsilon_{i}(\rho)$ denotes interaction energy of the Hall state and depends only on the particle density.

We are interested in the dispersion relation of the magneto-roton excitations. To this end, we linearize the equations of motion and turn on perturbations about the ground state:

$$
a_{\mu}=A_{\mu}+\tilde{a}_{\mu}, \quad \rho=\rho_{0}+\tilde{\rho}, \quad v_{i}=0+v_{i},
$$




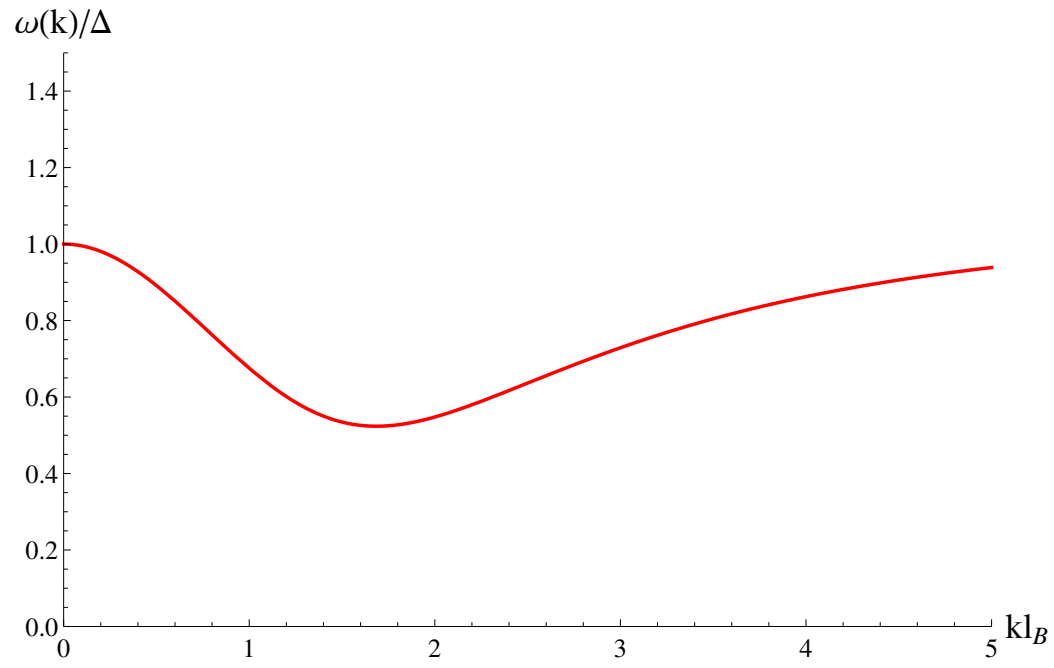

Figure 1. Dispersion relation of the collective mode with $\nu=1 / 3$ and $\lambda /\left(l_{B} \Delta\right)=0.3$. Minimum dispersion appears at $k_{\min } \approx 1.69 l_{B}^{-1}$.

where $\rho_{0}=\frac{\nu}{2 \pi} \epsilon^{i j} \partial_{i} A_{j}=\frac{\nu}{2 \pi} B$ is the ground state electron density and $\tilde{a}_{\mu}, \tilde{\rho}, v_{i}$ are small perturbations. We further assume that the energy arises purely from pairwise interactions with the form:

$$
\epsilon_{i}(\rho)=\frac{1}{2} \iint d^{2} x d^{2} y\left(\rho(x)-\rho_{0}\right) V(|\mathbf{x}-\mathbf{y}|)\left(\rho(y)-\rho_{0}\right) .
$$

For the sake of definiteness, we work with the Coulomb potential with a strength parameter $\lambda$ defined as:

$$
\frac{\lambda}{q}=\int d^{2}(\mathbf{x}-\mathbf{y}) V_{c}(|\mathbf{x}-\mathbf{y}|) e^{i(\mathbf{q}(\mathbf{x}-\mathbf{y}))} .
$$

It should be noted that even though the specifics of the calculation depend on the exact form of the chosen potential, the qualitative behavior that we derive here is independent of such details, so long as the potential remains repulsive.

In what follows we set $p=k l_{B}=k / \sqrt{B}$ and $\sigma_{s}=\frac{1}{2}(1-s)$. We note that the parameter $\alpha$ used in the non-universal functions $F$ and $G$ (see eq. (3.8)) is fixed by our first sum rule given in eq. (2.17): $\alpha=2 S_{4}$. Also, as noted previously, the inequality (2.26) is saturated in this model.

We find the dispersion relation of the magneto-roton mode to be:

$$
\omega(p)=\Delta \frac{\sqrt{1+2 \sigma_{s} p^{2}+\frac{\alpha \lambda}{\Delta l_{B}} p^{3}+\sigma_{s}^{2} p^{4}}}{1+\left(\alpha+\sigma_{s}\right) p^{2}},
$$

which exhibits the properties of the magneto-roton with a downward slope at small $p$ which turns around after a characteristic minimum (figure 1).

For the purpose of comparison, we also report this this dispersion relation up to fourth order in momentum expansion:

$$
\omega(p)=\Delta\left[1-2 S_{4} p^{2}+\frac{\lambda S_{4}}{l_{B} \Delta} p^{3}+\left(4 S_{4}^{2}+2 S_{4} \sigma_{s}\right) p^{4}+\mathcal{O}\left(p^{5}\right)\right] .
$$




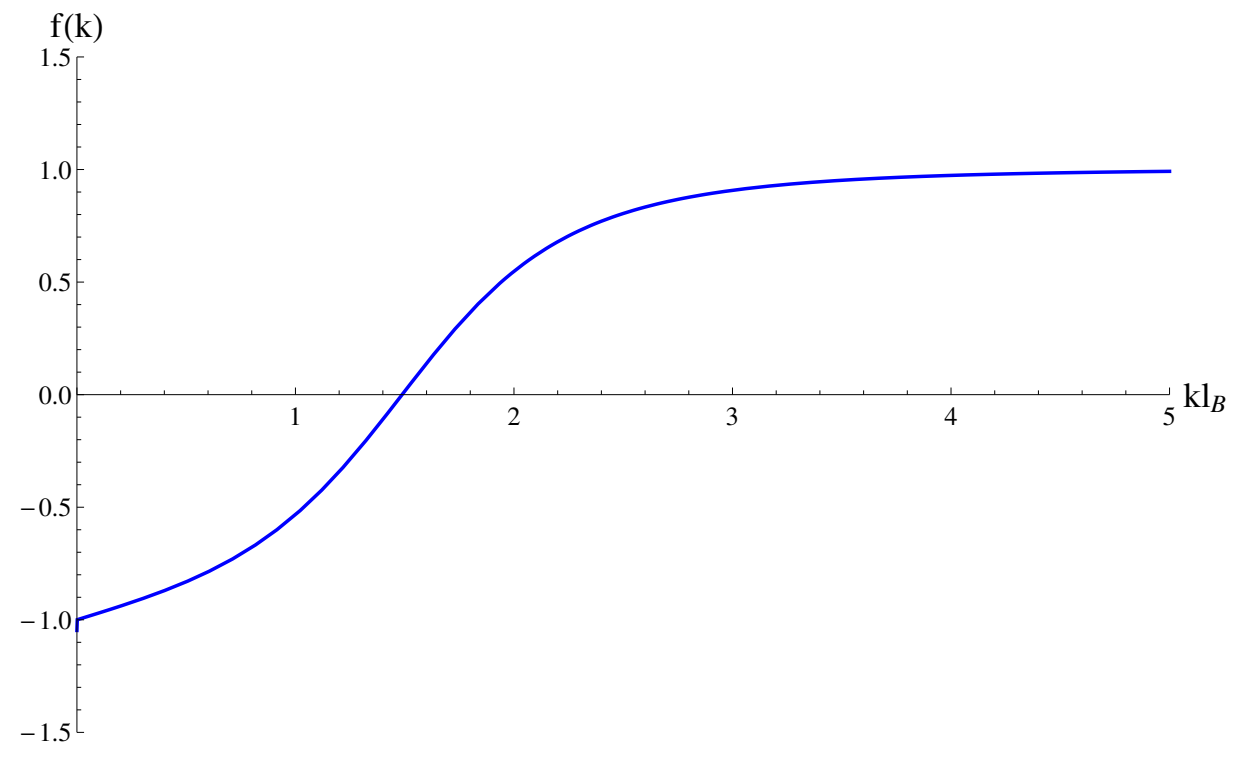

Figure 2. Polarization function $f(k)$, with $\nu=1 / 3, \lambda /\left(l_{B} \Delta\right)=0.3$. Linear polarization appears at $k \approx 1.49 l_{B}^{-1}$.

Note that the coefficient in front of $p^{2}$ is, for Laughlin's fractions, $-(1-\nu) / 4 \nu$, which is also seen in the model of ref. $[15,16]$. However, we cannot argue that this coefficient is universal. For example, the coefficient will change if we add to the action (3.7) a term proportional to the square of the spatial gradients of $\gamma_{i j}$.

There is one more interesting feature of this mode that reveals itself under closer inspection. From the equations of motion, we can derive that the eigenmodes satisfy:

$$
\mathbf{p} \times \mathbf{v}=i f(p) \mathbf{p} \cdot \mathbf{v}, \quad f(p)=\frac{1+p^{2} \sigma_{s}+\omega_{p}^{2} p^{2} G\left(\omega_{p}\right)}{\omega_{p} p^{2} F\left(\omega_{p}\right)},
$$

where $\mathbf{k} \times \mathbf{v}=\epsilon^{i j} k_{j} v_{j}$ and $\omega_{p}=\omega(p)$ given in (3.13).

If we decompose $\mathbf{v}$ into a parallel component $v_{\|}$and a perpendicular component $v_{\perp}$ to the direction of momentum $\mathbf{p}$, we find that $v_{\perp}=i f(p) v_{\|}$. Using the dispersion relation (3.13), and the explicit form of $G(\omega), F(\omega)$, we see that the value of $f(k)$ evolves from $f(0)=-1$ to $f\left(k \approx k_{\min }\right)=0$ and finally to $f(k=\infty)=1$ (figure 2 ).

This implies that, from the point of view of current pattern, the excitations exhibit counterclockwise rotation at small momenta, which turns into a linear oscillation in direction of $\mathbf{p}$ in the vicinity of the magneto-roton minimum and finally develops into clockwise rotation at large values of the momentum. It would be interesting to understand if this feature of the magneto-roton may be detected experimentally.

Here we only briefly discuss the implication of the model for the observation of the magneto-roton at low momentum in Raman scattering experiments. In previous theoretical treatments [23], it was assumed that the magneto-roton is excited chiefly through the coupling of electric field to density: $\rho E^{2}$. This coupling, however, implies that the intensity of the magneto-roton peak should scale as the fourth power of the magneto-roton momentum, $q^{4}$. In experiments, magneto-roton was seen down to even the lowest momenta [2], 
a fact that may be attributed to disorders violating momentum conservation. However, we cannot rule out a coupling of the type $T_{i j} E_{i} E_{j}$ from symmetry consideration, with $T_{i j}$ being the stress tensor. Even if the coefficient in front of this term is small, it would dominate the intensity of magneto-roton peak in the limit $q \rightarrow 0$, since the residue at the pole in in $\langle T T\rangle$ correlators remains finite in this limit. This coupling thus provides an alternative explanation of the observation of the magneto-roton at lowest momenta in Raman scattering experiments.

Moreover, in our model at $q=0$ the magneto-roton is circularly polarized with angular momentum 2. We suggest that the polarization of the magneto-roton at $q=0$ may be detectable by Raman scattering with polarized light.

\section{Conclusion}

In this paper we looked at gapped fractional quantum Hall states with filling factors $\nu<1$ in the regime where the Coulomb energy is much smaller than the cyclotron energy, however with energies comparable to that of the gap. We developed three sum rules involving the spectral densities of the stress tensor which we then used to verify Haldane's conjectured lower bound on the quartic coefficient of the structure factor $\mathcal{S}_{4}$, as well as introduce other inequalities.

We also introduced a simple model that saturates these inequalities via a mode which arises from the mixing between the oscillations of an internal metric and the hydrodynamic excitations. We identifed this mode as the magneto-roton and calculated its dispersion relation. We argued that the intensity of the magneto-roton line in Raman scattering experiments should not vanish at zero momentum, and that the magneto-roton at $q=0$ is a spin- 2 object. Finally, we suggest that the spin of the magneto-roton can be determined by Raman scattering with polarized light.

\section{Acknowledgments}

The authors thank Ilya Gruzberg, Michael Levin, Emil Martinec, Aron Pinczuk, and Paul Wiegmann for discussion. This work is supported, in part, by NSF MRSEC grant DMR0820054. D.T.S. is supported, in part, by DOE grant DE-FG02-90ER-40560 and a Simons Investigator grant from the Simons Foundation.

Open Access. This article is distributed under the terms of the Creative Commons Attribution License (CC-BY 4.0), which permits any use, distribution and reproduction in any medium, provided the original author(s) and source are credited.

\section{References}

[1] S.M. Girvin, A.H. MacDonald and P.M. Platzman, Magneto-roton theory of collective excitations in the fractional quantum Hall effect, Phys. Rev. B 33 (1986) 2481 [INSPIRE].

[2] A. Pinczuk, B. S. Dennis, L.N. Pfeiffer, and K. West, Observation of collective excitations in the fractional quantum Hall effect, Phys. Rev. Lett. 70 (1993) 3983. 
[3] M. Kang et al., Inelastic light scattering by gap excitations of fractional quantum Hall states at $1 / 3 \leq \nu \leq 2 / 3$, Phys. Rev. Lett. 84 (2000) 546.

[4] I.V. Kukushkin, J.H. Smet, V.W. Scarola, V. Umansky and K. von Klitzing, Dispersion of the excitations of fractional quantum Hall states, Science 324 (2009) 1044.

[5] C.F. Hirjibehedin et al., Splitting of long-wavelength modes of the fractional quantum Hall liquid at $\nu=1 / 3$, Phys. Rev. Lett. 95 (2005) 066803.

[6] F.D.M. Haldane, 'Hall viscosity' and intrinsic metric of incompressible fractional Hall fluids, arXiv:0906.1854 [INSPIRE].

[7] F.D.M. Haldane, Geometrical description of the fractional quantum Hall effect, Phys. Rev. Lett. 107 (2011) 116801 [arXiv:1106.3375] [INSPIRE].

[8] B. Yang, Z.-H. Hu, Z. Papić and F.D.M. Haldane, Moddel wavefunctions for the collective modes and the magneto-roton theory of the fractional quantum Hall effect, Phys. Rev. Lett. 108 (2012) 256807 [arXiv: 1201.4165].

[9] X.G. Wen and A. Zee, Shift and spin vector: new topological quantum numbers for the Hall fluids, Phys. Rev. Lett. 69 (1992) 953 [Erratum ibid. 69 (1992) 3000] [INSPIRE].

[10] D.T. Son, Newton-Cartan geometry and the quantum Hall effect, arXiv:1306.0638 [INSPIRE].

[11] P. Wiegmann, Quantum hydrodynamics of fractional Hall effect: quantum Kirchhoff equations, arXiv:1211.5132.

[12] A.G. Abanov, On the effective hydrodynamics of the fractional quantum Hall effect, J. Phys. A 46 (2013) 292001 [INSPIRE].

[13] B. Bradlyn, M. Goldstein and N. Read, Kubo formulas for viscosity: Hall viscosity, Ward identities and the relation with conductivity, Phys. Rev. B 86 (2012) 245309 [arXiv: 1207.7021] [INSPIRE].

[14] J.E. Avron, R. Seiler and P.G. Zograf, Viscosity of quantum Hall fluids, Phys. Rev. Lett. 75 (1995) 697 [INSPIRE].

[15] I.V. Tokatly, Unified hydrodynamics theory of the lowest Landau level, Phys. Rev. B 74 (2006) 035333 [cond-mat/0512706].

[16] I.V. Tokatly, Magnetoelasticity theory of incompressible quantum Hall liquids, Phys. Rev. B 73 (2006) 205340 [cond-mat/0505715].

[17] I.V. Tokatly and G. Vignale, New collective mode in the fractional quantum Hall liquid, Phys. Rev. Lett. 98 (2007) 026805 [cond-mat/0607705].

[18] R.Z. Qiu, F.D.M. Haldane, X. Wan, K. Yang and S. Yi, Model anisotropic quantum Hall states, Phys. Rev. B 85 (2012) 115308 [arXiv:1201.1983].

[19] F. D. M. Haldane, Self-duality and long-wavelength behavior of the Landau-level guiding-center structure function, and the shear modulus of fractional quantum Hall fluids, arXiv: 1112.0990.

[20] N. Read and E.H. Rezayi, Hall viscosity, orbital spin and geometry: paired superfluids and quantum Hall systems, Phys. Rev. B 84 (2011) 085316 [arXiv: 1008.0210] [INSPIRE].

[21] S. Dubovsky, T. Gregoire, A. Nicolis and R. Rattazzi, Null energy condition and superluminal propagation, JHEP 03 (2006) 025 [hep-th/0512260] [INSPIRE]. 
[22] J. Maciejko, B. Hsu, S.A. Kivelson, Y. Park and S.L. Sondhi, Field theory of the quantum Hall nematic transition, Phys. Rev. B 88 (2013) 125137 [arXiv:1303.3041] [INSPIRE].

[23] P.M. Platzman and S. He, Resonant Raman scattering from mobile electrons in the fractional quantum Hall regime, Phys. Rev. B 49 (1994) 13674. 